\title{
Ice Particle Interarrival Times Measured with a Fast FSSP
}

\author{
P. R. Field, R. WoOd, AND P. R. A. BRown \\ Met Office, Farnborough, Hampshire, United Kingdom \\ P. H. Kaye, E. Hirst, and R. Greenaway \\ STRC, University of Hertfordshire, Hatfield, Hertfordshire, United Kingdom \\ J. А. Sмітн \\ Met Office, Farnborough, Hampshire, United Kingdom
}

(Manuscript received 16 April 2002, in final form 2 August 2002)

ABSTRACT

\begin{abstract}
Ice particle interarrival times have been measured with a fast forward scattering spectrometer probe (FSSP). The distribution of interarrival times is bimodal instead of the exponential distribution expected for a Poisson process. The interarrival time modes are located at $\sim 10^{-2}$ and $\sim 10^{-4} \mathrm{~s}$. This equates to horizontal spacings on both the centimeter and meter scales. The characteristics of the interarrival times are well modeled by a Markov chain process that couples together two independent Poisson processes operating at different scales. The possibility that ice crystals shattering on the probe tip causes the bimodal interarrival times is explored and cannot be ruled out. If the observations are indicating real spacings of particles in clouds, then the observations show very localized (centimeter scale) concentrations of $\sim 100 \mathrm{~s} \mathrm{~cm}^{-3}$ embedded within an average concentration of typically $\sim 1 \mathrm{~cm}^{-3}$. If the localized high concentrations are produced by the ice crystals shattering, then the concentration measured by the FSSP is overcounted by a factor of 5 in the worst case measured here, but more typically by a factor of 2 . This uncertainty in concentration will adversely affect the predicted radiative influence of these clouds.
\end{abstract}

\section{Introduction}

Airborne probes capable of detecting large numbers of ice crystals, such as Particle Measuring Systems (PMS) 2D probes and the Stratton Park Engineering Company Cloud Particle Imager, encounter problems when attempting to detect and classify particles with diameters comparable to the probe resolution. Other probes, such as the Cloudscope and replicator-based detectors, provide higher resolution but require laborious analysis and have a relatively small sample volume. In an effort to overcome these difficulties various researchers have attempted to make use of the forward scattering spectrometer probe (FSSP) for investigating ice crystals. However, some workers have felt that the FSSP is overcounting in ice clouds. Gardiner and Hallett (1985) suggested that the FSSP gives a false response in ice clouds and should not be used for characterization of small ice crystals. Since then Gayet et al. (1996) compared the PMS cloud probe (2D-C) and FSSP size distributions

Corresponding author address: Dr. P. R. Field, Met Office, Building Y46, Cody Technology Park, Ively Road, Farnborough, Hampshire, GU14 OLX, United Kingdom.

E-mail: paul.field@metoffice.com in the poorly measured overlap region and proposed that observations made with the FSSP probe can be believed if the ice crystals are small and spherical, and in such cases there is good agreement between the 2D-C and FSSP. Poor agreement was generally found between the 2D-C and FSSP if the 2D-C spectrum was broad.

Recently, there has been interest in analyzing the spatial structure of liquid phase clouds at centimeter scales or less (e.g., Brenguier 1993; Pinsky and Khain 1996; Chaumat and Brenguier 2001). It has been postulated that the effects of droplet inertia in a turbulent velocity field might lead to changes in the supersaturation field and hence changes in the droplet growth rates. These same effects could also be occurring in glaciated cloud. In addition, other sources of small ice particles such as secondary ice multiplication (Hallett-Mossop process) and evaporative breakup, observed by Bacon et al. (1998) in the laboratory, could result in small-scale spatial structure in ice cloud.

In this paper we will try to determine whether the fast FSSP can be used in glaciated clouds as a tool for studying ice particle spatial distributions at the millimetric scale required to investigate the effects of secondary multiplication, evaporative breakup, and tur- 




FIG. 1. (left) Schematic of standard and modified inlet fitted to FFSSP. (right) Picture of modified (silver) and unmodified (black) FFSSP inlets.

bulence. The following sections describe the instrument itself, the characteristics of its data in water and ice clouds, and a statistical model to help interpret the latter. We then describe variations of the parameters of this model with environmental variables such as temperature and discuss the implications in terms of different mechanisms that may be responsible for the observations.

\section{Fast FSSP description and response to liquid water cloud}

The "fast" probe used is an FSSP-100 used to detect spherical particles with a $1-22.5-\mu \mathrm{m}$ radius with modified electronics to allow logging of particle arrival time, pulse size, annulus signal, and pulse duration, time stamped with a temporal resolution of $8.3 \times 10^{-8} \mathrm{~s}$ for each particle. The dead time of $6 \mu$ s per particle for a standard FSSP has been eliminated [e.g., see Brenguier et al. (1998) for a description of similar fast FSSP system].

The optical arrangement is as described in Dye and Baumgardner (1984) for a standard FSSP. The pulse size is the maximum scattering signal recorded by the detection diode $\left(3^{\circ}-12^{\circ}\right.$ forward scattering angle) for each particle. The annulus signal is the maximum signal recorded by the annulus diode $\left(12^{\circ}-15^{\circ}\right)$ and in combination with the pulse size determines whether or not the detected particle was in the depth of field of the FSSP. The pulse duration is the length of time that the pulse signal remained greater than a predefined threshold value for a given particle and is a measure of how long a particle takes to transit across the FSSP sample volume. Because the FSSP laser beam cross section is nominally circular, particles that cross the beam near the center will have a longer pulse duration than those particles that cross near the beam's edge.

For one of the aircraft flights (A815), a modification was made to the FSSP inlet to investigate the possibility that the inlet geometry could affect the FSSP response. Figure 1 schematically depicts the modified and standard FSSP inlet side by side. The main difference is that the tube has been removed. This now places the exposed optical arms upstream of the sample volume.

Before we proceed to analyze the response of the fast FSSP (FFSSP) to ice particles we will first illustrate the FFSSP's behavior in liquid cloud. Brenguier (1993) showed that interarrival times between droplets in stratocumulus cloud were modeled well by a Poisson process. Although the droplet counting is a Poisson process, the droplet mean arrival time varies in time and space due to turbulence in clouds, resulting in a generalized or inhomogeneous Poisson process (Snyder and Miller 1991), as described by Pawlowska et al. (1997).

For a Poisson process, the probability of observing an event in a time period short enough for only one event to be observed, $\delta t$, is

$$
P_{1}=\frac{\delta t}{\tau}
$$

where $1 / \tau$ is the mean arrival rate of the Poisson process. The probability of not observing an event in the same time period is then

$$
P_{0}=1-P_{1}=1-\frac{\delta t}{\tau} .
$$

We are interested in the distribution of particle interarrival times, $\Delta t$, that is, the time interval between one event and the next. We can compute the probability of having to wait a given interarrival time for an event by considering $n$ short periods of time, $\delta t$, during which no events are observed followed by one short period during which an event is observed. Because the chance of an event occurring or not occurring in each time period is independent, the probabilities are compounded to give

$$
P(\Delta t+\delta t)=\left(1-\frac{\delta t}{\tau}\right)^{n} \frac{\delta t}{\tau} .
$$

For large $n,(1-\delta t / \tau)^{n}=\exp (-n \delta t / \tau)$, and setting $\Delta t$ $=n \delta t$ gives

$$
\frac{P(\Delta t+\delta t)}{\delta t}=\frac{1}{\tau} \exp \left(-\frac{\Delta t}{\tau}\right) .
$$

As the short time interval considered for the observation of single events becomes small, $\delta t \rightarrow 0$, Eq. (4) can then be written as

$$
\frac{d P(\Delta t)}{d t}=\frac{1}{\tau} \exp \left(-\frac{\Delta t}{\tau}\right)
$$

Integrating Eq. (5) gives the probability of finding interarrival times greater than $\Delta t_{i}$ : yielding an exponential function

$$
P\left(\Delta t>\Delta t_{i}\right)=\int_{\Delta t_{i}}^{\infty} \frac{d P(\Delta t)}{d t}=\exp \left(-\frac{\Delta t_{i}}{\tau}\right),
$$

where $\Delta t_{i}$ is the interarrival time considered. 



FIG. 2. (a) Cumulative interarrival time frequency for a 0.1 -s interval in stratocumulus cloud. (b) The same data as in (a) but plotted on a semilog plot. The vertical dotted line is the mean interarrival time, and the thin-lined histogram is the Poisson distribution with the same mean as the data. (c) The cumulative $\chi^{2}$ distribution for 7 degrees of freedom. The solid line is the theoretical curve. The open squares are from flight A767, and the plus signs are from flight A766.

Brenguier (1993) and Paluch and Baumgardner (1989) have shown, using Eq. (6), that interarrival times are exponentially distributed and that the Poisson rate can be estimated from the slope of the cumulative frequency distribution. In the subsequent analysis for ice cloud we will be interested in the behavior of the interarrival times over several orders of magnitude, necessitating the use of the semilogarithmic plot. To maintain an equal area-equal probability property we will use

$$
\frac{d P(\Delta t)}{d \ln t}=\frac{\Delta t}{\tau} \exp \left(-\frac{\Delta t}{\tau}\right)
$$

This function is modal with a peak at $\Delta t=\tau$, indicating that $\tau$ is an estimate of the mean interarrival time. By dividing observed interarrival times into discrete classes in logarithmic space we can use Eq. (7) to estimate the expected number of occurrences in each class for a homogeneous Poisson process and hence compute $\chi^{2}$, given that we know $\tau$. Values of $\chi^{2}$ computed for many sets of interarrival times should follow the $\chi^{2}$ distribution.

By minimizing $\chi^{2}$ to find $\tau$, best-fit Poisson functions were generated for observations from two flights (A766, A767) in liquid phase stratocumulus cloud around the British Isles. The interarrival times were analyzed in 0.1 -s intervals over $100 \mathrm{~s}$. The cumulative frequency of each interarrival time for one 0.1-s interval is shown in Fig. 2a and displays the exponential behavior expected for a Poisson process. Figure $2 \mathrm{~b}$ shows the logarithmically binned version of the same data and the bestfit Poisson distribution obtained after a $\chi^{2}$ minimization process. This particular interval has a $\chi^{2}$ value of 6.9 for 7 degrees of freedom. Combining a large number of the $\chi^{2}$ values allows us to generate a cumulative distribution for $\chi^{2}$ that can be compared with the $\chi^{2}$ values obtained from tables (e.g., Snedecor and Cochran 1967). In Fig. 2c the tabulated values for 7 degrees of freedom and cumulative frequencies obtained from the two stratocumulus datasets are compared. The curves show good agreement with the tabulated values, confirming the validity of the Poisson assumption on these scales $(10 \mathrm{~m})$ for these two flights in liquid phase clouds.

The number of droplets encountered is usually $\sim 1000$ times greater than the number of ice particles for the same interval of time. This means that in the subsequent analysis of ice crystals we will not have the luxury of being able to select a small enough time interval to avoid the influence of large-scale effects.

\section{Response of the FFSSP in ice cloud}

The data presented are from six flights carried out around the British Isles. All of the data are taken from straight and level runs in ice cloud. The cloud conditions sampled are as follows.

- A800, 13 October 2000: A cirrus layer ahead of a warm front encroaching from the west was sampled from near cloud top at $-42^{\circ} \mathrm{C}(9400 \mathrm{~m})$ down to $-23^{\circ} \mathrm{C}(7000 \mathrm{~m})$, which was still in cloud.

- A801, 16 October 2000: Cirrostratus was sampled just behind an occluded front running down the center of the United Kingdom. Cloud top was at $-41^{\circ} \mathrm{C}(8800$ $\mathrm{m})$, and cloud base was at $-22^{\circ} \mathrm{C}(5800 \mathrm{~m})$.

- A802, 17 October 2000: Cirrostratus ahead of a warm front moving in from the west was sampled between cloud top at $-48^{\circ} \mathrm{C}(9600 \mathrm{~m})$ and cloud base at $-39^{\circ} \mathrm{C}$ $(8300 \mathrm{~m})$.

- A803, 20 October 2000: Runs were carried out in a mixed phase layer of cloud ahead of a frontal system approaching from the southwest. The layer cloud sampled was dominated at one end by ice and at the other by supercooled water at $-15^{\circ} \mathrm{C}(5500 \mathrm{~m})$ but only regions devoid of liquid were used.

- A806, 21 November 2000: Runs were made deep within a frontal cloud between $-16^{\circ} \mathrm{C}(5000 \mathrm{~m})$ and $-6^{\circ} \mathrm{C}(3000 \mathrm{~m})$.

- A815, 19 February 2001: Altostratus layered ice cloud was sampled with a modified FSSP inlet at $-26^{\circ} \mathrm{C}$ $(6000 \mathrm{~m})$ for these runs.

Examples are presented in Figs. 3-5 of the response of the FFSSP in ice cloud. These examples are typical of what is observed and are shown to indicate the variation in the degree of bimodality seen in the interarrival time histogram. The Small Ice Detector (SID) probe (Hirst et al. 2001) was used to identify runs or parts of 

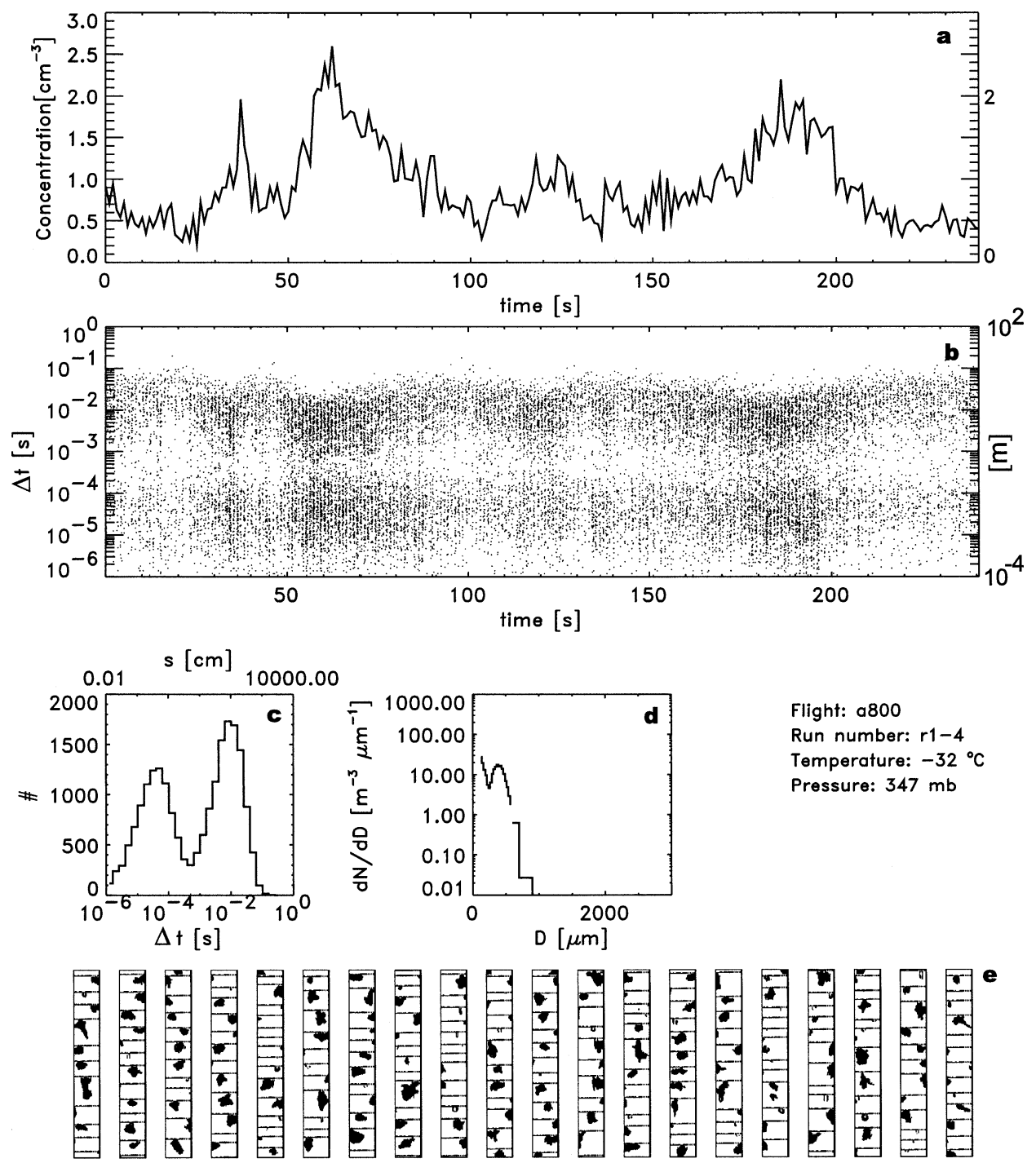

FIG. 3. Composite example plot from an aircraft run in glaciated cloud for flight A800 run 1.4: (a) the measured concentration $(1 \mathrm{~Hz})$ from the FFSSP as a function of run time; (b) the interarrival times as a function of run time for each particle; (c) a histogram of the interarrival times for the data shown in (b); (d) the combined 2D-C and 2D-P size spectra; (e) example images from the 2D-C probe as a function of time; i.e., the horizontal position relates to the run time given in (a) and (b). The width of each image bar is $800 \mu \mathrm{m}$.

runs in which the liquid phase was absent for the analysis. We used contiguous data, so the sections of the run either up to when the last liquid water was observed or after the first liquid water was observed were used. Figures 3-5 each consist of five panels: (a) indicates the $1-\mathrm{Hz}$ particle concentration measured by the FFSSP as a function of aircraft time; (b) shows the interarrival time (interarrival distance is given on the right for a nominal airspeed of $100 \mathrm{~m} \mathrm{~s}^{-1}$ ) for each particle as a function of time; (c) is a histogram of the interarrival times in the panel above; (d) is the average 2D-C (nominal particle size range: $25-800 \mu \mathrm{m}$ ) and the PMS precipitation probe (2D-P; nominal particle size range: 200-6400 $\mu \mathrm{m}$ ) size distribution for the period consid- ered; and (e) shows 2D-C imagery from the aircraft run as a function of time.

In the examples shown the FFSSP concentration varies between 0.1 and $15 \mathrm{~cm}^{-3}$. The interarrival times show a distinct bimodal nature with modes at $\approx 10^{-2}$ and $\approx 10^{-4} \mathrm{~s}$, which is equivalent to spacings of $\approx 100$ and $\approx 1 \mathrm{~cm}$ if the particles were distributed in this way in the atmosphere. The relative contribution to the total concentration from the modes varies from flight to flight. Generally, as the concentration decreases the mode of the maximum interarrival time mode moves to longer interarrival times, whereas the shorter interarrival time mode remains more constant.

To confirm that the FFSSP observations of bimodal 



FIG. 4. The same as Fig. 3 but for flight A802 run 3.3.

interarrival time were not an artifact of the electronics employed, the Small Ice Detector was modified to allow monitoring of the particle detection times with a fast oscilloscope. This indicated that particles were also arriving in the SID sample volume with bimodal interarrival times.

\section{Modeling the observations with a Markov chain process}

When interpreting the liquid droplet interarrival times the a priori assumption of a Poisson process was made. Here we will introduce a model that will aid in the interpretation of the ice particle data. Like the assumption made in liquid water cloud of the validity of a Poisson process the choice here of statistical model does not provide an explanation for the physical cause of the observed distribution.
A homogeneous Poisson process is an inadequate fit to the ice cloud interarrival time. What is required is a model of an inhomogeneous Poisson process that reproduces a distinct bimodal interarrival time distribution. To reproduce the observations of particles arriving in the sample volume, use is made of a Markov chain that contains three states (Fig. 6). The process is memoryless, and the outcome of the current action depends only on the current state. The Markov chain allows us to compound two Poisson processes to represent the arrival of individual particles at two different scales. States 1 and 2 are "waiting states," when no particle is observed during the interval, while state 0 represents the observation of a particle. For a Poisson process the chance of observing an event in an interval, $\delta t$, is $\delta t / \tau$, where $1 / \tau$ is the mean arrival rate for the Poisson process. Conversely, the chance of not observing an event is simply $1-\delta t / \tau$. Figure 6 shows the three states linked 



FIG. 5. The same as Fig. 3 but for flight A803 run 2.3.

by arrows that are annotated with their respective probabilities of occurence. So for state 1 the arrow returning directly to state 1 represents the situation in which no event is observed $\left(1-\delta t / \tau_{1}\right)$, and the arrow linking state 1 to state 0 represents the observation of an event $\left(\delta t / \tau_{1}\right)$ for a Poisson process with mean arrival rate

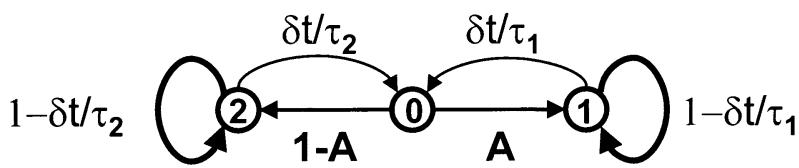

FIG. 6. A three-state Markov chain diagram. The numbers in the circles represent the three states. State 0 is the presence of a particle, and states 1 and 2 indicate the absence of a particle. The arrows show the possible ways of moving between states. The terms next to the arrows indicate the probability of a state change occurring via that route. It can be seen that it is not possible to go from state 1 directly to state 2 ; the route, instead, has to be through the intermediate state 0 .
$1 / \tau_{1}$. Similarly, state 2 represents a Poisson process with mean arrival rate $1 / \tau_{2}$. When state 0 is attained the system returns to state 1 or 2 , according to the weighting of $A$ and $(1-A)$, respectively. More formally, the probabilities given in Fig. 6 can be represented with the following transition matrix:

$$
\mathbf{P}=\left(\begin{array}{lll}
p_{00} & p_{01} & p_{02} \\
p_{10} & p_{11} & p_{12} \\
p_{20} & p_{21} & p_{22}
\end{array}\right)=\left(\begin{array}{ccc}
0 & A & 1-A \\
\frac{\delta t}{\tau_{1}} & 1-\frac{\delta t}{\tau_{1}} & 0 \\
\frac{\delta t}{\tau_{2}} & 0 & 1-\frac{\delta t}{\tau_{2}}
\end{array}\right) .
$$

The probability matrix can be used to generate the probability of occurrence of any event described by the system. The probability of going from state 0 to 1 and back to 0 is 


$$
p_{010}=p_{01} p_{10}=A \frac{\delta t}{\tau_{1}} .
$$

The probability of going from state 0 to 1 and then remaining in state 1 for $n$ time intervals before going to 0 is

$$
p_{01 \cdots 10}=p_{01} p_{11}^{n} p_{10}=A \frac{\delta t}{\tau_{1}}\left(1-\frac{\delta t}{\tau_{1}}\right)^{n} .
$$

Similarly, we can write

$$
p_{02 \cdots 20}=(1-A) \frac{\delta t}{\tau_{2}}\left(1-\frac{\delta t}{\tau_{2}}\right)^{n}
$$

for state 2 . The overall probability of reaching state 0 after $n$ intervals in either state 1 or 2 is then just

$$
\begin{aligned}
p(\Delta t+\delta t) & =p_{01 \cdots 10}+p_{02 \cdots 20} \\
& =A \frac{\delta t}{\tau_{1}}\left(1-\frac{\delta t}{\tau_{1}}\right)^{n}+(1-A) \frac{\delta t}{\tau_{2}}\left(1-\frac{\delta t}{\tau_{2}}\right)^{n}
\end{aligned}
$$

Letting interarrival time $\Delta t=n \delta t$ and, for large $n$, (1 $\left.-\Delta t / \tau_{1}\right)^{n}=\exp \left(-t / \tau_{1}\right)$ we obtain

$$
\begin{aligned}
\frac{p(\Delta t+\delta t)}{\delta t} & =\lim _{\delta t \rightarrow 0} \frac{d p(\Delta t)}{d t} \\
& =A \frac{1}{\tau_{1}} \exp \left(-\frac{\Delta t}{\tau_{1}}\right)+(1-A) \frac{1}{\tau_{2}} \exp \left(-\frac{\Delta t}{\tau_{2}}\right)
\end{aligned}
$$

Hence, the probability of finding interarrival times, $\Delta t$, between $\Delta t_{i}$ and $\infty$ is $\int_{\Delta t_{i}}^{\infty} p(t) d t$ :

$$
P\left(\Delta t>\Delta t_{i}\right)=A \exp \left(-\frac{\Delta t}{\tau_{1}}\right)+(1-A) \exp \left(-\frac{\Delta t}{\tau_{2}}\right)
$$

which reduces to the expected result for a Poisson process if $A$ is set to unity [cf. Eq. (6)]. The magnitude of $(1-A)$ tells us the contribution to the total number of particles counted by the FSSP made by the particles associated with $\tau_{2}$. Hence, $(1-A)$ can be used as a measure of the degree of clustering if we define those particles associated with state transitions involving state 2 as being clustered.

We will now assume that $\tau_{1}>\tau_{2}$, and whenever state 0 is arrived at from state 2 the observed particle (state 0 ) is part of a cluster of particles that are bunched in groups of 1 to many particles. To define the clustering threshold we consider the particles related to the state 2 process and an interarrival time that only a small proportion $(0.02)$ of these particles have,

$$
\frac{P\left(\Delta t_{\text {thresh }}, \infty\right)}{P\left(\tau_{2}, \infty\right)}=\exp \left(1-\frac{\Delta t_{\text {thresh }}}{\tau_{2}}\right)=\exp (-4)
$$

and, therefore, $\Delta t_{\text {thresh }}=5 \tau_{2}$. In addition, if $5 \tau_{2}>\tau_{1}$, then the distribution will not be considered bimodal. Unfortunately, using a threshold means that some of the particles associated with state 1 will also be labeled as clustered particles (typically 5\%), but this does not overly affect our results. When we consider a cluster consisting of a train of particles that have interarrival times less than $\Delta t_{\text {thresh }}$, we can define the arrival time of the cluster as that of the first particle in the train. When we look at the distribution of the clusters by considering the interarrival times of the clusters, we find that they are distributed randomly, as suggested by the Markov chain process. It should be mentioned here that other authors (e.g., Kostinski and Jameson 1997) have introduced definitions for describing the degree of clustering. These definitions rely on the fact that the Poisson process intensity is being modulated by an underlying larger-scale process that introduces a variation of the interarrival time statistical properties. In this case the random particle counting process is an inhomogeneous Poisson process, and the probability distribution of the interarrival times is represented by Eq. (14) rather than a single exponential distribution characteristic of a homogeneous Poisson process.

Having developed this model as a potential means of describing the observations, we then need a method to estimate the parameters $A, \tau_{1}$, and $\tau_{2}$ from the data. We employed a two-stage $\chi^{2}$ minimization technique. One thousand initial guesses for the three parameter values were randomly chosen and the $\chi^{2}$ for each guess was computed. After this stage, another 1000 random guesses were made in smaller ranges around the best-guessed values from the previous stage. The best values after the second stage were taken as the fitted parameters. This two-stage process was repeated 10 times for each interval and showed that the fit was robust. Once $\tau_{1}$ and $\tau_{2}$ were estimated, it was then possible to obtain pulse durations and pulse size values (the peak scattering intensity for each particle) for populations of particles close to the two interarrival time modal values. Because ice concentrations are much lower than droplet concentrations, sampling over longer time periods is required to accumulate sufficient numbers of particles for analysis. We have chosen a 10-s interval for the present analysis. Unfortunately, this means that larger-scale effects may alter the simple inhomogeneous Poisson model of the particle spacing suggested by the Markov chain model outlined above. In practice this effect was reflected in higher $\chi^{2}$ values than would be expected, but assessment of the fits using the $\chi^{2}$ minimization technique indicated that the automatic method was estimating parameter values accurately. Figure 7 shows the result of the fitting technique applied to the synthetic data that was generated with the Markov chain model for the following parameters: $A=0.70 ; \tau_{1}=0.010 ; \tau_{2}$ $=0.00010$. For this case the estimated values obtained from the minimization of $\chi^{2}$ were $A=0.70, \tau_{1}=$ 

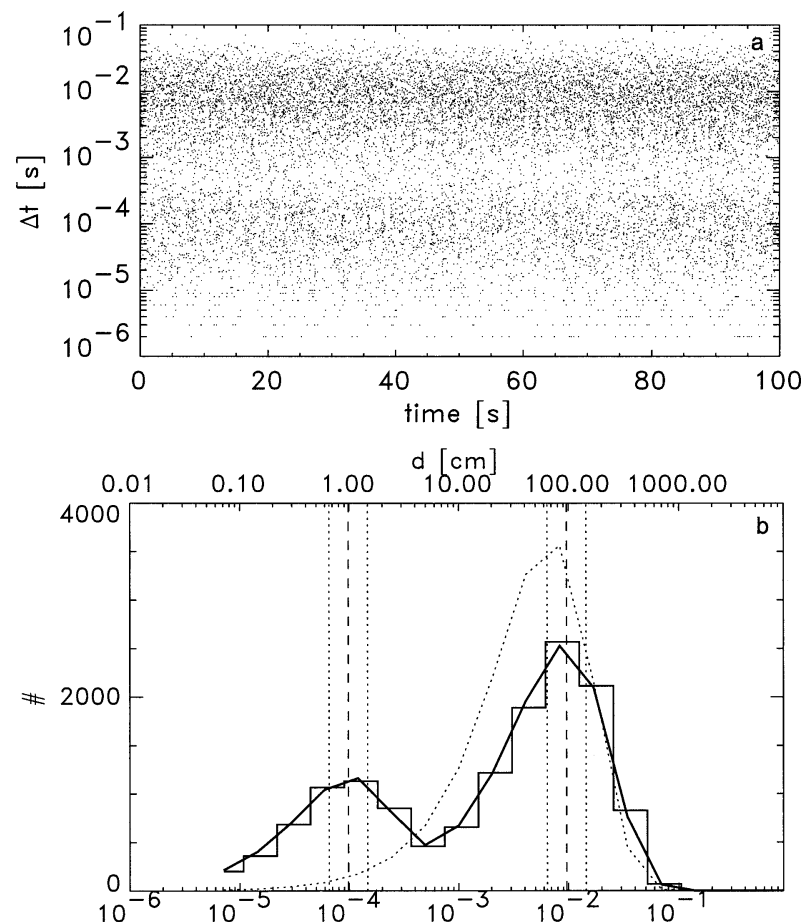

FIG. 7. (a) A synthetic time series of interarrival times generated from the Markov chain model with parameters $A=0.7, \tau_{1}=10^{-2}$, and $\tau_{2}=10^{-4}$. (b) Histogram of the interarrival times with the bestfit function (solid line) obtained from the minimization of $\chi^{2}$. The vertical dashed lines indicate the values of $\tau_{1}$ and $\tau_{2}$. The vertical dotted lines bracket the values of interarrival time used to compare populations of particles associated with the different modes. The scale at the top indicates the equivalent interparticle spacing for a nominal TAS of $100 \mathrm{~m} \mathrm{~s}^{-1}$. The dotted curve indicates the equivalent Poisson curve for the overall average interarrival time.

0.0099 , and $\tau_{2}=0.00010$, which compare well with the values used to generate the dataset.

This simple Markov chain model allows some characteristics of the clustering to be described. The probability for a cluster to contain $n$ particles is proportional to the probability of transferring from state 0 to $2, n$ times consecutively [i.e., $(1-A)^{n}$ ]. Thus, the expected number of clusters of size $n\left[N_{c}(n)\right]$ relative to clusters of another size, $m\left[N_{c}(m)\right]$ say, is

$$
\frac{N_{c}(n)}{N_{c}(m)}=\frac{(1-A)^{n}}{(1-A)^{m}} .
$$

If this ratio is plotted as a function of $n$ on a $\log$-linear plot, a straight line with a gradient of $\log (1-A)$ is the result:

$$
\log \left[\frac{N_{c}(n)}{N_{c}(m)}\right]=(n-m) \log (1-A) .
$$

Because $(1-A)<1$, the gradient is always negative, and clusters formed from increasingly greater numbers of particles become less frequent. To compare the predicted clustering behavior with the observed data we use an interarrival time threshold to differentiate be-
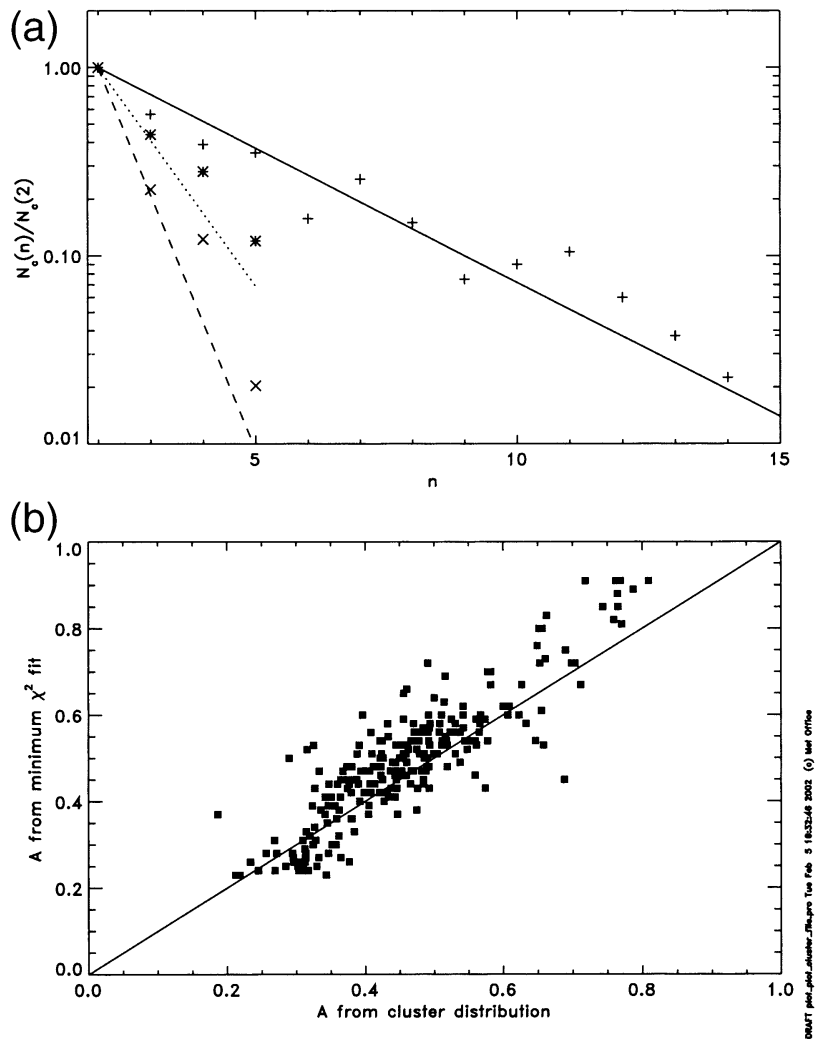

FIG. 8. (a) The ratio of the number of clusters composed of $n$ particles compared to the number of cluster formed of two particles. The * are from flight A800, the + are from flight A806, and the $\times$ are from flight A801. The straight lines are the predicted cluster distributions based on an estimate of $A$ obtained from the $\chi^{2}$ fitting. (b) A scatterplot of $A$ estimated from the $\chi^{2}$ fitting and $A$ estimated from fitting least squares exponential functions to the distributions in (a).

tween particles belonging to clusters and those that do not, as described above. The Markov chain model provides an independent method to estimate $A$ using the distribution of clusters. We can compare this value with that obtained from the $\chi^{2}$ fitting to assess the applicability of the model. Figure 8 a shows cluster distribution ratios $\left[N_{c}(n) / N_{c}(m)\right]$ as a function of cluster size for three 10 -s intervals from different flights. The straight lines are the predicted cluster distributions based on the estimate of $A$ from the $\chi^{2}$ fit. The predicted lines appear to match quite well with the observed cluster distribution. Figure $8 \mathrm{~b}$ shows a comparison of $A$ estimated from least squares fits to the cluster distribution compared to the $\chi^{2}$ estimate of $A$. The straight line represents a 1:1 fit and suggests that the Markov chain model can provide a robust estimate of this parameter.

\section{Relationships between Markov chain parameters and environmental variables}

Using the $\chi^{2}$ minimization technique described in section 4, the Markov chain parameters have been es- 
TABLE 1. Percentile values of parameters obtained from minimum $\chi^{2}$ fit to observed interarrival time distributions, where $p(\Delta t)=A\left(1 / \tau_{1}\right) \exp \left(-\Delta t / \tau_{1}\right)+(1-A) 1 / \tau_{2} \exp \left(-\Delta t / \tau_{2}\right)$.

\begin{tabular}{|c|c|c|c|c|c|c|c|c|c|}
\hline \multirow[b]{2}{*}{ Flight } & \multicolumn{3}{|c|}{$A$} & \multicolumn{3}{|c|}{$\tau_{1}(\mathrm{~ms})$} & \multicolumn{3}{|c|}{$\tau_{2}(\mu \mathrm{s})$} \\
\hline & 25 th & 50th & 75 th & 25 th & 50 th & 75 th & 25 th & 50 th & 75th \\
\hline A 800 & 0.53 & 0.57 & 0.63 & 18 & 29 & 48 & 63 & 74 & 89 \\
\hline A801 & 0.48 & 0.56 & 0.68 & 13 & 18 & 32 & 81 & 90 & 100 \\
\hline A802 & 0.80 & 0.87 & 0.89 & 36 & 52 & 59 & 150 & 210 & 2700 \\
\hline A803 & 0.41 & 0.49 & 0.56 & 8 & 10 & 15 & 61 & 72 & 77 \\
\hline A806 & 0.32 & 0.42 & 0.48 & 9 & 12 & 14 & 64 & 70 & 76 \\
\hline A815 & 0.80 & 0.85 & 0.88 & 19 & 27 & 38 & 110 & 120 & 140 \\
\hline
\end{tabular}

timated for $10-\mathrm{s}$ intervals from each flight. Estimates are made for regions containing ice crystals only, as determined by asphericity measurements from the SID.

Table 1 contains 25 th, 50 th, and 75 th percentile values for the minimum $\chi^{2}$ fitted parameters for the individual flights. Figures $9-11$ are plots of the minimum $\chi^{2}$ fitted parameters and environmental variables.

Figure 9a shows a plot of $A$ versus the average environmental temperature for the run. If A815 is ignored, the data display an ordered behavior with temperaturehigh $A$ values at cold temperatures decreasing to an approximately constant value of $0.4-0.5$ at temperatures warmer than $-30^{\circ} \mathrm{C}$. The data from the modified inlet flight (A815) have greater values of $A$ compared to data from the other flights in the same temperature range. Importantly, some points from A803 lie above the main body of data at $-13^{\circ} \mathrm{C}$. These points come from the early part of a run, when the concentrations are relatively low, and 2D-C imagery shows a larger proportion of small particles compared to other periods on this flight.

Figure $9 \mathrm{~b}$ shows that there is a similar correlation, but of opposite sign, between A and true air speed (TAS) when TAS is used rather than temperature. As the TAS increases so does $A$. This is partly to be expected when operating at higher altitudes (colder temperatures), where the aircraft is normally operating at a higher TAS. Again the data points from A815 and several from A803 can be seen above the main body of the data at 125 and $117 \mathrm{~m} \mathrm{~s}^{-1}$, respectively.

Figures $9 \mathrm{c}$ and $9 \mathrm{~d}$ show $A$ versus $\tau_{1}$ and $A$ versus $\tau_{2}$, respectively. In contrast to $\tau_{1}$, which displays no correlation with $A, \tau_{2}$ displays a positive correlation with $A$. In this figure the data from A815 lie coincident with the data from the other flights. Figure 9d suggests that as the clustering becomes more pronounced (decreasing A) $\tau_{2}$ and, hence, the interparticle spacing decrease.

In Fig. 10a $A$ is plotted against the quotient of 2D$\mathrm{C}$ concentration for particles larger than $350 \mu \mathrm{m}$ and concentration of 2D-C particles larger than $100 \mu \mathrm{m}$. This ratio is a measure of how broad the ice particle size distribution is. Higher values of this ratio indicate the presence of relatively more larger particles. This figure indicates that values of $A>0.8$ coincide with narrow 2D-C size distributions. Again, A815 is an ex- ception, displaying high $A$ values even when larger ice particles are present.

A plot of the normalized difference between the average pulse duration taken by the particle with interarrival times close to the modal values as a function of temperature is shown in Fig. 10b. For interarrival times within a factor of 1.5 of the long interarrival time mode, the pulse durations were averaged to give $\operatorname{tr}_{1}$. This was done for the short interarrival mode as well, to give $\operatorname{tr}_{2}$. The first point about this plot is that the difference in pulse duration can be both positive and negative, implying that clustered particles can be traveling both faster and slower than the unclustered particles. Second, the difference tends to be positive at temperatures colder than $-20^{\circ} \mathrm{C}$.

A plot of $A$ as a function of the dissipation rate, $\epsilon$ (Fig. 10c), indicates that turbulence appears to have no obvious effect upon the amount of clustering observed. Bacon et al. 1998 and Dong et al. (1994) have observed the breakup of dendritic ice crystals when evaporating, leading to the production of small fragments. It has been suggested that small-scale turbulence may be responsible for large variations in relative humidity, which may lead to the evaporative breakup of ice crystals, for instance. Such a mechanism would be expected to produce clusters of particles and result in bimodal interarrival times. To investigate this possible mechanism we have looked at dissipation rates estimated from $32-\mathrm{Hz}$ data. Dissipation rates are estimated using the $32-\mathrm{Hz}$ vertical and horizontal winds following the method of Kaimal (1973). This involves estimation of the second-order velocity structure function, which is related to the dissipation rate via the Kolmogorov "two-thirds" law. Dissipation rate is a more effective measure of small-scale turbulence than the wind variances because wind variances can include nonturbulent wave motions. The lack of correlation between the dissipation rate and the degree of clustering suggests that turbulence is not playing a role in controlling the observed clustering.

Finally, we present a figure showing the concentration of particles measured by the FFSSP as a function of temperature for particles in both modes (Fig. 11a) and for particles in the long interarrival time mode (Fig. 11b). The first figure shows concentrations in the range $0.1-5 \mathrm{~cm}^{-3}$, with a tendency for higher concentrations 

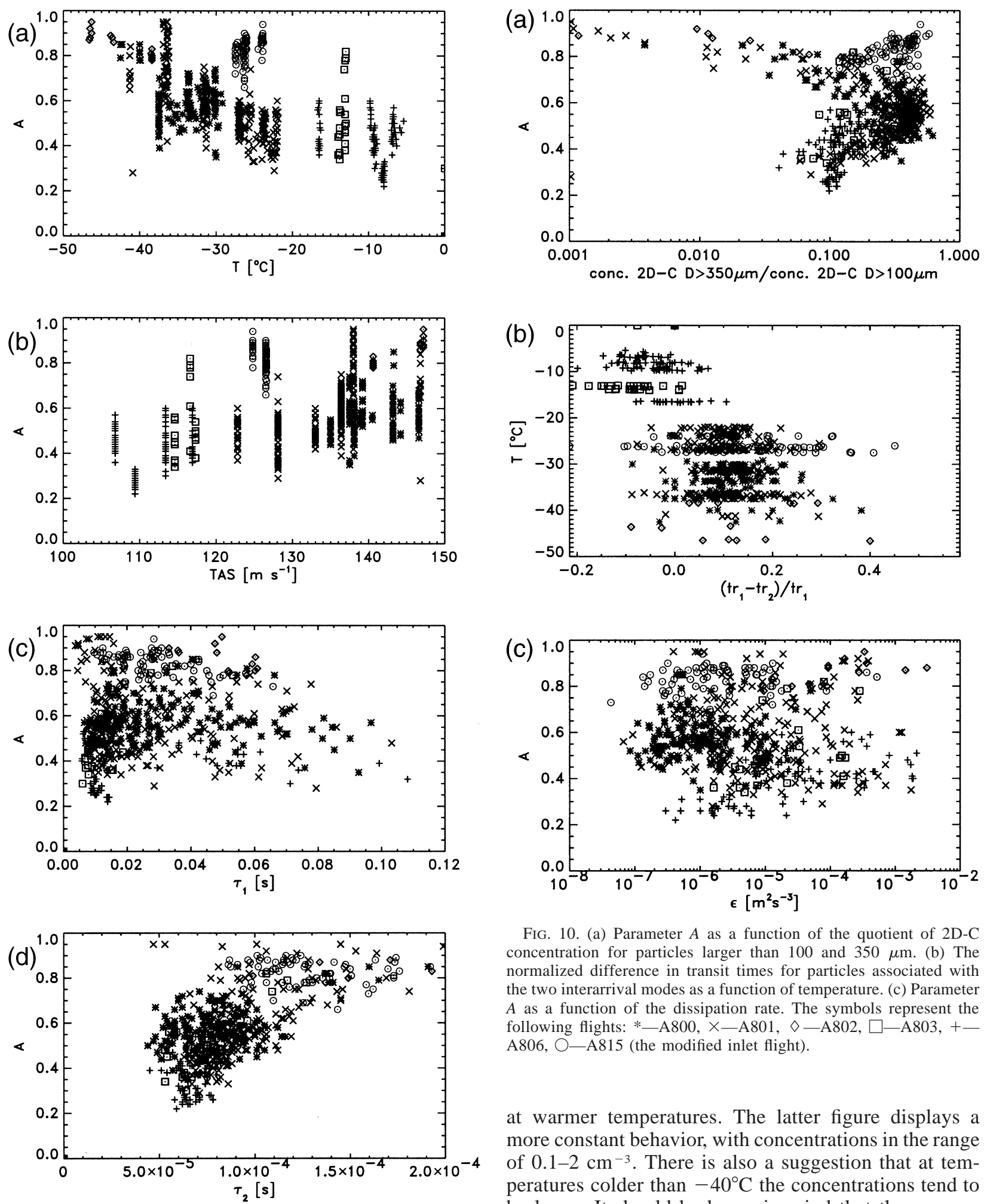

FIG. 10. (a) Parameter $A$ as a function of the quotient of 2D-C concentration for particles larger than 100 and $350 \mu \mathrm{m}$. (b) The normalized difference in transit times for particles associated with the two interarrival modes as a function of temperature. (c) Parameter $A$ as a function of the dissipation rate. The symbols represent the following flights: *-A800, $\times$-A801, $\diamond-\mathrm{A} 802, \square-\mathrm{A} 803,+-$ A806, $\bigcirc-A 815$ (the modified inlet flight).

at warmer temperatures. The latter figure displays a more constant behavior, with concentrations in the range of $0.1-2 \mathrm{~cm}^{-3}$. There is also a suggestion that at temperatures colder than $-40^{\circ} \mathrm{C}$ the concentrations tend to be lower. It should be borne in mind that the concentrations are estimated assuming that the sample volume for droplets is applicable to ice crystals. This is not necessarily the case, as particle size and habit may affect the depth of field. Another problem with the estimate of the sample volume is the situation in which large (b) Parameter $A$ as a function of TAS. (c) Parameter $A$ as a function of parameter $\tau_{1}$. (d) Parameter $A$ as a function of parameter $\tau_{2}$. The symbols represent the following flights: *-A800, $\times-\mathrm{A} 801, \diamond-$ A802, $\square$-A803, + -A806, O-A815 (the modified inlet flight). 

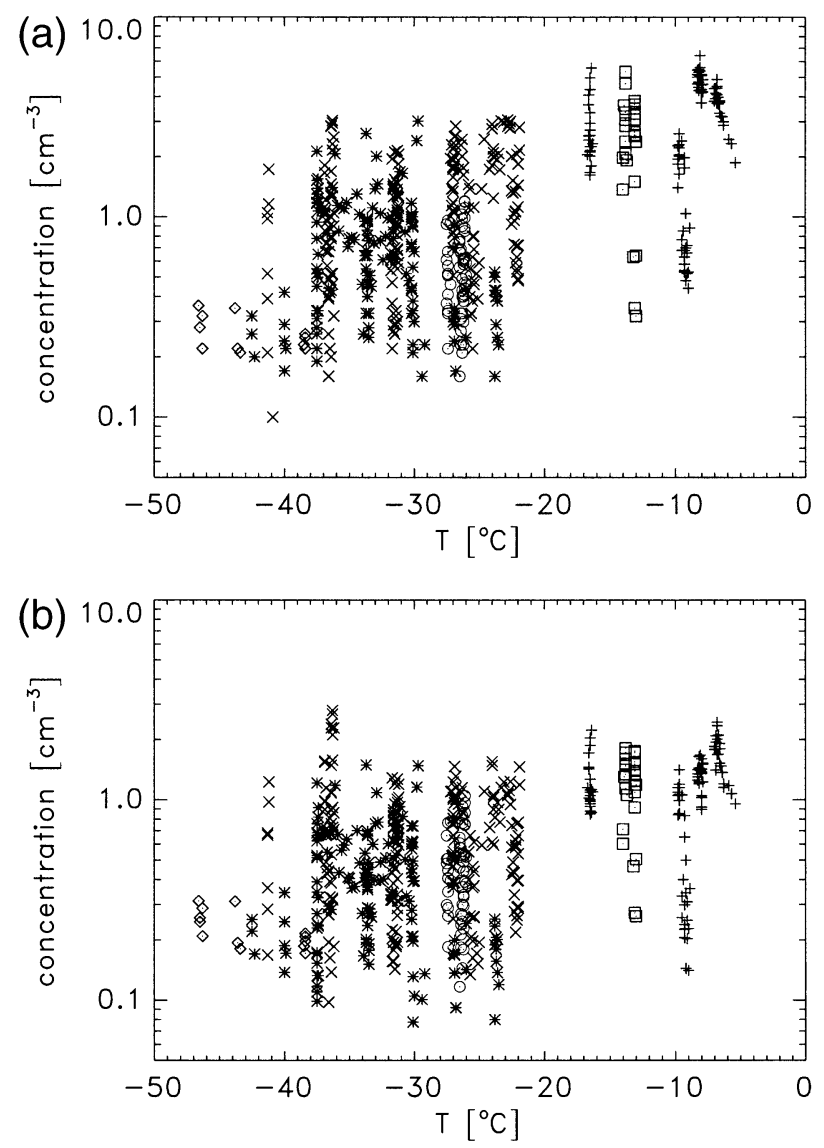

FIG. 11. (a) FFSSP concentration as function of temperature. (b) FFSSP concentration in the long interarrival time mode as function of temperature. The symbols represent the following flights: *A800, $メ$ A801, $\diamond-A 802, \square-A 803,+-A 806$, O-A815 (the modified inlet flight).

crystals just touch the edge of the sample volume. This has the effect of artificially enhancing the concentration of the large particles. This multiplying effect is linear with particle size, but, as the concentrations of large particles typically fall off in an exponential manner, the contribution to the FFSSP concentration from 2D sized particles is small-typically less than $10 \%$ for the data presented here.

\section{Physical origin of bimodal interarrival times}

The Markov chain model is able to describe the response of the FFSSP to ice, but it does not provide an explanation for the physical mechanism responsible. Scenarios to be considered are (i) ice crystal breakup on the probe, or in the airflow of the FFSSP upstream of its sample region; and (ii) clustered spacing of ice particles in the atmosphere due to real microphysical processes. We now examine a number of characteristics of the data in an attempt to distinguish between the two processes.

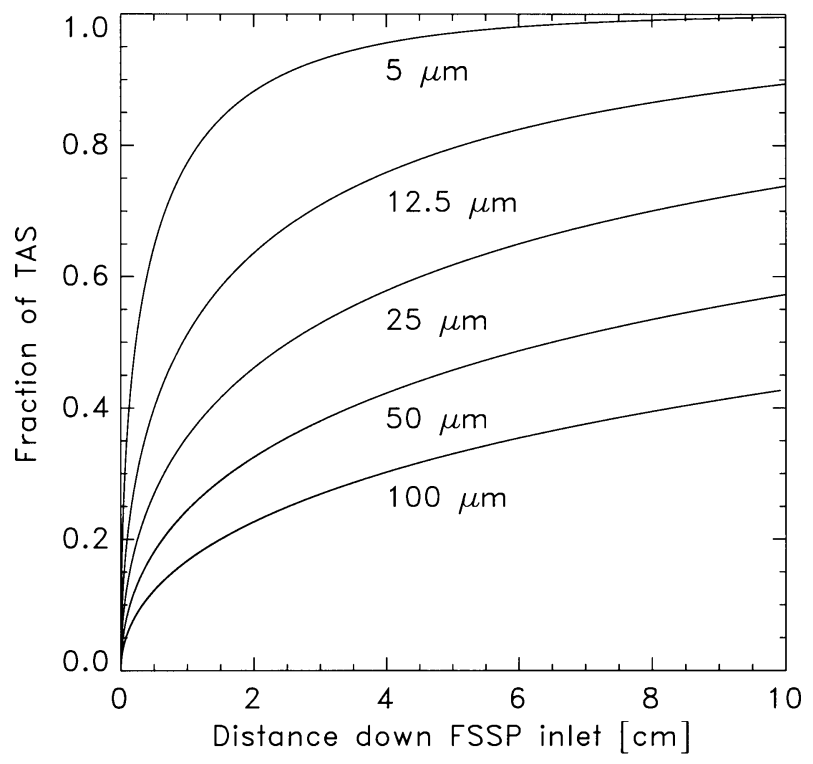

FIG. 12. Speed reached by an ice sphere accelerated from rest in an airflow with a speed equal to the TAS as a function of distance traveled. The length of the FFSSP inlet tube is $10 \mathrm{~cm}$.

If shattering of particles on the probe housing were responsible, then we may postulate that the debris would travel through the sample volume at a lower speed than the unaffected particles. We can examine this point with a simple model that is set in the rest frame of the probe. Ice spheres are accelerated from rest in an airflow that has a velocity equal to the true air speed, over a distance of $10 \mathrm{~cm}$ (the distance from the tip of the sampling tube to the scattering volume in a standard FSSP). Figure 12 shows the velocity of ice spheres (as a fraction of the TAS) as a function of the distance they have traveled. The figure indicates that fragments smaller than $25 \mu \mathrm{m}$ in radius starting at rest will accelerate to at least $70 \%$ of the true air speed. In practice the ice crystals have larger drag coefficients, and any debris will not necessarily be starting from rest, so it seems unlikely that there will be a large difference in the speeds of debris and unaffected particles as they enter the sample volume. Hence, we cannot use transit times as a test of whether or not shattering is occurring. A second question that could be asked about the shattering scenario is: can debris cross from the edge of the inlet tube to the sample volume? Using the same aerodynamic model, critical trajectories were computed for debris particles that start with a speed along the axis of the inlet tube equal to the TAS and an initial perpendicular launch velocity into the center of the tube that would transport the particles from the probe tip to the sample volume. It can be seen from Fig. 13 that apart from the smallest particles (radius $<10 \mu \mathrm{m}$ ) debris particles only require a small fraction of the TAS in the perpendicular direction to fall into the sample volume. Based on this simple model it would seem unwise to rule out the pos- 


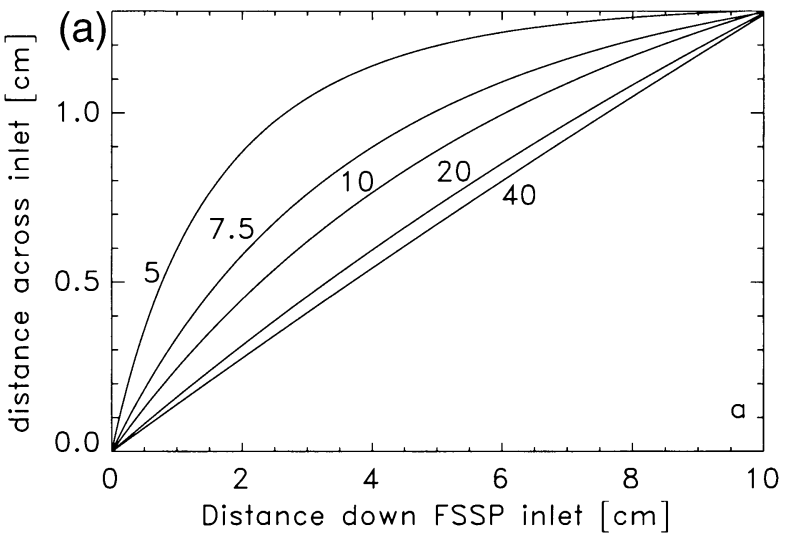

(b)

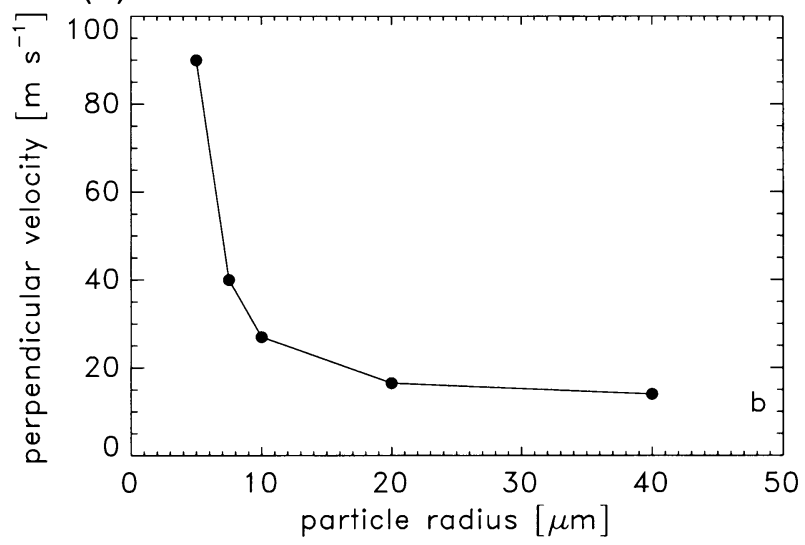

FIG. 13. (a) Critical trajectories of ice spheres launched perpendicularly from the edge of the inlet tube toward the center. The upper right corner of the plot represents the FFSSP sample volume. The numbers next to the lines indicate the particle radius in microns. (b) The perpendicular launch velocity required for ice spheres of various sizes to follow the critical trajectories shown in (a).

sibility that shattering may affect the FFSSP measurements.

Another simple model to consider for the shattering scenario is the relationship between the geometry of the inlet tube and sample volume. For the unmodified FFSSP the inlet tube has a 5 -mm-wide, $45^{\circ}$ inward facing bevel where the debris could be envisaged to originate. Of course, the debris production region could be either larger or smaller than this. Assuming that the production zone is a $5-\mathrm{mm}$ annulus with an inside diameter of $25 \mathrm{~mm}$ means that the ratio of this production zone to the FFSSP sample volume is of the order of $10^{4}$. In a replicator study Hallett (1976) reports that ice crystals larger than a few hundred (100-300) micrometers in diameter will shatter on impact with a formvarcovered surface. It is probably safe to assume that the same could happen when large ice crystals come into contact with the FFSSP probe tip. Therefore, we can estimate a shattering efficiency based upon the relative concentrations of large ice crystals (concentration of particles larger than $\left.350 \mu \mathrm{m} ; C_{D>350}\right)$ and the concen-

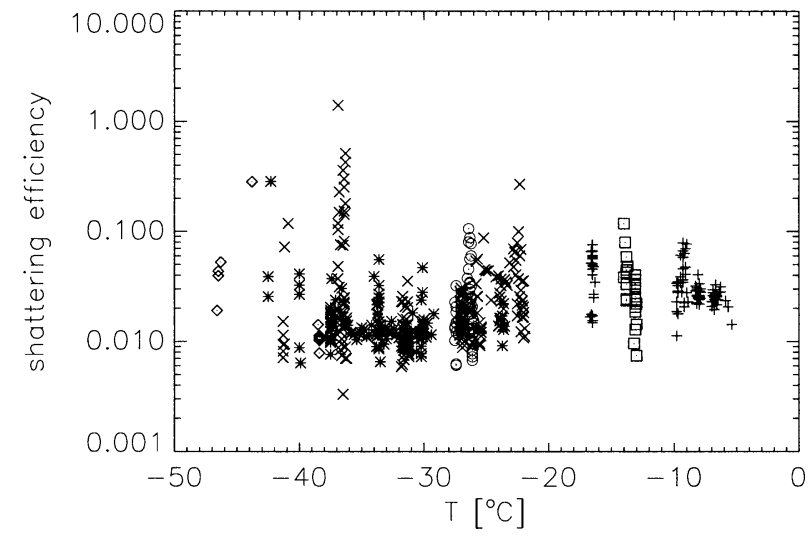

FIG. 14. Shattering efficiency as a function of temperature. The symbols represent the following flights: *-A800, $\times$-A801, $\diamond-$ A802, $\square$-A803, +-A806, ○-A815 (the modified inlet flight).

tration of clusters $\left(C_{\mathrm{cl}}\right)$. The concentration of clusters is given by

$$
C_{\mathrm{cl}}=\frac{C_{\mathrm{FFSSP}}(1-A)}{\bar{n}},
$$

where $C_{\mathrm{FFSSP}}$ is the measured FFSSP concentration, (1 $A$ ) is the fraction of clustered particles, and $\bar{n}$ is the average number of particles in a cluster. It can be shown that the average number of particles in each cluster, $\bar{n}$, is given by $-1 / \ln (1-A)$. Hence, a shattering efficiency, $E_{s}$, can then be defined as the ratio of concentration of clusters, $C_{\mathrm{cl}}$ to the large crystal concentration, $C_{D>350}$ with a correction included for the difference in sample volumes of the FFSSP and the annulus of the inlet tube:

$$
E_{s}=\frac{C_{\mathrm{FFSSP}}(1-A)[-\ln (1-A)]}{C_{D>350} \times 10^{4}} .
$$

The numerator represents the concentration of clusters of particles, that is, each cluster is a single unit. The denominator represents the maximum concentration of clusters that may be produced on the assumption that every ice crystal larger than $350 \mu \mathrm{m}$ would produce a single cluster event and that smaller crystals would produce none. If values of shattering efficiency greatly exceed unity then we can rule out the hypothesis that the clustered particles result from shattering. A plot of the shattering efficiency (Fig. 14) shows values in the range 0.01-0.1 for most cases apart from flight A801, which has much higher values $(0.01-1.0)$. It is interesting to note that the modified inlet flight seems to have the same shattering efficiency as the other flights.

\section{Discussion and summary}

In this section we will provide arguments for and against the ice crystal shattering and natural crystal bunching hypotheses. The main evidence for the shattering hypothesis is provided by the temperature versus $A$ plot (Fig. 9a), the difference in pulse duration plot 
(Fig. 10b), and the 2D-C concentration versus $A$ plot (Fig. 10a). In these three figures the data obtained using the modified FSSP inlet lie outside of the main body of data obtained from the other flights. If evaporative breakup were really occurring, then the average particle transit time for particles with interarrival times close the the modal values would be the same. Figure 10b shows that this is not the case. However, the difference is not always of the same sense. For this observation we have no explanation.

Evidence against the shattering hypothesis is found in the shattering efficiency plot (Fig. 14), which shows the modified inlet data lying within the main body of data in contrast to the data depicted in Figs. 9a and 10a. The modified inlet does present surfaces upstream from the sample volume, on which particle shattering may be occurring. Nevertheless, it may be expected that such a significant change in the intake design would produce an observable difference in the shattering efficiency.

Direct evidence against the evaporative breakup hypothesis is seen in the dissipation rate, $\epsilon$ versus $A$ plot (Fig. 10c). A correlation between these variables might have been expected if turbulence were producing smallscale variations in the local humidity field and more intense turbulence were generating greater variation. These variations would lead to regions of subsaturation with respect to ice that could drive the evaporative breakup of large ice crystals to produce smaller particles. The lack of correlation implies that the intensity of the turbulence is not controlling the degree of clustering.

It appears that we have no conclusive evidence to rule out the possibility that shattering of ice crystals may be taking place on the housing of the probe and affecting the observations. We suggest that to determine the importance of shattering an experiment needs to be undertaken that involves flying two FFSSPs-one with a modified inlet - on the same aircraft in glaciated clouds. Even if shattering is occurring the greatest contribution we have observed from the flights presented is $1-A$ $=0.8$, and more usually it is $1-A<0.6$. This means that in the worst case the concentration is overestimated by a factor of 5 but is more likely to be a factor of 2 or less. Obviously such large variations in concentration estimates can have a large impact upon the subsequent prediction of ice cloud radiative properties.

To summarize, the particle interarrival times measured by an FFSSP in ice clouds are bimodal with modes at $10^{-2}$ and $10^{-4} \mathrm{~s}$. The bimodal nature of the interarrival times is well described with a Markov chain model that incorporates two separate Poisson processes with rates reflecting the observed modal values. It is difficult to determine the physical origin of the bimodality, and because of this more observations need to be carried out. The role of shattering should not be ruled out. However, if shattering is responsible for the bimodality the measured FSSP concentrations will be overestimated by a factor of 5 at most. If the particles within a cluster are ignored then the concentrations measured by the FSSP typically fall in the range of $0.1-1 \mathrm{~cm}^{-3}$. If the clustered particles are included then FSSP concentrations can reach $6 \mathrm{~cm}^{-3}$ on average, but locally of the order of $100 \mathrm{~cm}^{-3}$ in the frontal cloud measured. If the clustering is real then a microphysical mechanism will need to be invoked to explain the observed localized high concentrations of $\sim 100 \mathrm{~cm}^{-3}$ over centimeter spatial scales.

Acknowledgments. We would like to thank Jean-Louis Brenguier for detailed comments and suggestions that greatly improved the quality of this paper. Some of the data used were collected as part of the NERC, CWVC thematic program.

\section{REFERENCES}

Bacon, N. J., B. D. Swanson, M. B. Baker, and E. J. Davis, 1998: Breakup of levitated frost particles. J. Geophys. Res., 103 (D12), $13763-13775$.

Brenguier, J.-L., 1993: Observations of cloud microstructure at the centimeter scale. J. Appl. Meteor., 32, 783-793.

_ - T. Bourrianne, A. De Araujo Coelho, J. Isbert, R. Paytavi, D. Trevarin, and P. Weschler, 1998: Improvements of droplet size distribution measurements with the Fast-FSSP. J. Atmos. Oceanic Technol., 15, 1077-1090.

Chaumat, L., and J.-L. Brenguier, 2001: Droplet spectra broadening in cumulus clouds. Part II: Microscale droplet concentration heterogeneities. J. Atmos. Sci., 58, 642-654.

Dong, Y., R. G. Oraltay, and J. Hallett, 1994: Ice particle generation during evaporation. Atmos. Res., 32, 45-53.

Dye, J. E., and D. Baumgardner, 1984: Evaluation of the Forward Scattering Spectrometer Probe. Part I: Electronic and optical studies. J. Atmos. Oceanic Technol., 1, 329-344.

Gardiner, B. A., and J. Hallett, 1985: Degradation of in-cloud forward scattering spectrometer probe measurements in the presence of ice particles. J. Atmos. Oceanic Technol., 2, 171-180.

Gayet, J.-F., G. Febvre, and H. Larsen, 1996: The reliability of the PMS FSSP in the presence of small ice crystals. J. Atmos. Oceanic Technol., 13, 1300-1310.

Hallet, J., 1976: Measurement of size, concentration and structure of atmospheric particulates by the airborne continuous particle replicator. Tech. Rep. AFGL-TR-76-0419, Air Force Geophysics Laboratory, Air Force Systems Command, United States Air Force, Hanscom AFB, MA, 92 pp.

Hirst, E., P. H. Kaye, R. S. Greenaway, P. R. Field, and D. W. Johnson, 2001: Discrimination of micrometre-sized ice and super-cooled droplets in mixed-phase cloud. Atmos. Environ., 35, 33-47.

Kaimal, J. C., 1973: Turbulence spectra, length scales and structure parameters in the stable boundary layer. Bound.-Layer Meteor., 4, 289-309.

Kostinski, A. B., and A. R. Jameson, 1997: Fluctuation properties of precipitation. Part I: On deviations of single-size drop counts from the Poisson distribution. J. Atmos. Sci., 54, 2174-2186.

Paluch, I. R., and D. G. Baumgardner, 1989: Entrainment and finescale mixing in a continental convective cloud. J. Atmos. Sci., 46, 261-278.

Pawlowska, H., J.-L. Brenguier, and G. Salut, 1997: Optimal nonlinear estimation for cloud particle measurements. J. Atmos. Oceanic. Technol., 14, 88-104.

Pinsky, M. B., and A. P. Khain, 1996: Simulations of drop fall in a homogeneous isotropic turbulent flow. Atmos. Res., 40, 223-259.

Snedecor, G. W., and W. G. Cochran, 1967: Statistical Methods. 6th ed. The Iowa State University Press, 593 pp.

Snyder, D. L., and M. J. Miller, 1991: Random Point Processes in Time and Space. Springer-Verlag, $480 \mathrm{pp}$. 